\title{
SOME REMARKS ON THE MECHANISMS AT WORK IN THE BETA CANIS MAJORIS STARS*
}

\author{
P. LEDOUX \\ Institut d'Astrophysique, Cointe-Sclessin, Belgium \\ Received April 30, 1958
}

\section{ABSTRACT}

It is shown that van Hoof's suggestion concerning the broadening of the lines associated with one of the components in the variation of the $\beta$ Canis Majoris stars cannot work in the case of purely radial oscillations. Some remarks on other hypotheses, such as that of Struve and Odgers, and on non-radial oscillations are added.

\section{INTRODUCTION}

In a recent paper van Hoof (1957) has summarized anew the most typical characteristics of the $\beta$ Cephei stars and has proposed a new interpretation in terms of two radial oscillations having markedly different effects on the macroturbulence in the external layers.

Although van Hoof's paper introduces an interesting point of view, some of his suggestions deserve some further comments, and some are misleading. First, a remark on notation. One of the most intriguing properties of many of these stars is the presence in their variations of two close periods, often denoted by $P_{1}$ and $P_{2}$. This, however, makes it awkward to find a distinct notation for the different theoretical modes of linear oscillation, and here we shall call the two observed periods $P$ and $P^{\prime}$, the prime denoting the oscillation associated with a broadening of the lines.

\section{ON THE BEHAVIOR OF THE TWO OSCILlations $P$ AND $P^{\prime}$ IN THE EXTERNAL LAYERS}

It has been well known since the work of Miss Kluyver (1936) that the occurrence of nearly equal periods $P$ and $P^{\prime}$ or frequencies $\sigma$ and $\sigma^{\prime}$ in a non-linear oscillation may be due to the coupling between two linear modes with frequencies $\sigma_{\mu}$ and $\sigma_{\nu}$ presenting a close two-to-one commensurability:

$$
\sigma_{\nu}=2 \sigma_{\mu}+\epsilon \quad(\epsilon \text { very small }) .
$$

In this context it is worth recalling the complete form of the corresponding solution. Let $\xi$ denote the relative displacement $\delta r / r_{0}$. If $\xi$ can be treated as infinitely small, the problem may be linearized, and it admits an infinite discrete set of eigen-values, $\sigma_{i}$, and orthogonal eigen-solutions, $\xi_{i}$, corresponding to the different modes of radial oscillations. However, if the finite character of $\xi$ is taken into account, it must then satisfy a non-linear partial differential equation of the second order in the time and the radius, which, in general, is not separable. One may then attempt a solution by a series expansion,

$$
\xi=\sum_{i} \xi_{i}\left(r_{0}\right) g_{i}(t)
$$

where the $\xi_{i}\left(r_{0}\right)$ 's are the linear eigen-solutions referred to above and the $g_{i}(t)$ 's are functions of the time to be determined.

* Published with the aid of a grant from the American Astronomical Society. 
Usually, only two terms are kept in equation (2), say those corresponding to $i=\mu$ and $i=\nu$, so that

$$
\xi=\xi_{\mu}\left(r_{0}\right) g_{\mu}(t)+\xi_{\nu}\left(r_{0}\right) g_{\nu}(t),
$$

and, in the partial differential equation, only terms up to the second degree in $\xi$ are retained. In that case, neglecting further the terms in $g_{\nu}^{2}$, it can be shown (Rosseland 1949) that, if one adopts as a first approximation for the $g$ 's

$$
\begin{aligned}
& g_{\mu, 1}=\cos \sigma_{\mu} t, \\
& g_{\nu, 1}=\cos \left(\sigma_{\nu} t-\Phi\right),
\end{aligned}
$$

the second approximation is given by

$$
\begin{aligned}
g_{\mu, 2}=A_{\mu} \cos \left(\sigma_{\mu} t+\Psi_{\mu}\right)+ & \frac{E \cos \left(\sigma_{\mu} t-\Phi\right)}{4 \sigma_{\mu}\left(\sigma_{\nu}-2 \sigma_{\mu}\right)}-\frac{D}{6 \sigma_{\mu}^{2}} \cos 2 \sigma_{\mu} t \\
& -\frac{E}{2} \frac{\cos \left[\left(\sigma_{\nu}-\sigma_{\mu}\right) t-\phi\right]}{\sigma_{\nu}\left(\sigma_{\nu}-2 \sigma_{\mu}\right)}-\frac{E}{2} \frac{\cos \left[\left(\sigma_{\mu}+\sigma_{\nu}\right) t-\Phi\right]}{\sigma_{\nu}\left(2 \sigma_{\mu}+\sigma_{\nu}\right)}
\end{aligned}
$$

and

$$
\begin{aligned}
g_{\nu, 2}=A_{\nu} \cos \left(\sigma_{\nu} t+\Psi_{\nu}\right)-\frac{d}{2} & \frac{\cos \sigma_{\nu} t}{\left(\sigma_{\nu}-2 \sigma_{\mu}\right)\left(\sigma_{\nu}+2 \sigma_{\mu}\right)}+\frac{d}{2} \frac{\cos 2 \sigma_{\mu} t}{\left(\sigma_{\nu}-2 \sigma_{\mu}\right)\left(\sigma_{\nu}+2 \sigma_{\mu}\right)} \\
& +\frac{e}{2} \frac{\cos \left[\left(\sigma_{\nu}-\sigma_{\mu}\right) t-\Phi\right]}{\sigma_{\mu}\left(2 \sigma_{\nu}-\sigma_{\mu}\right)}-\frac{e}{2} \frac{\cos \left[\left(\sigma_{\mu}+\sigma_{\nu}\right) t-\Phi\right]}{\sigma_{\mu}\left(2 \sigma_{\nu}+\sigma_{\mu}\right)}
\end{aligned}
$$

where, contrary to Rosseland, we have isolated explicitly the terms with frequencies $\sigma_{\mu}$ and $\sigma_{\nu}$ (second terms in eqs. [5] and [6]) amplified by subharmonic resonance. The exact values of the constants $A_{\mu}, \Psi_{\mu}, A_{\nu}$, and $\Psi_{\nu}$ have no importance here, and the parameters $E$ and $e$ characterize the mutual coupling of the modes $\xi_{\mu}$ and $\xi_{\nu}$, while $D$ and $d$ characterize the self-coupling of $\xi_{\mu}$. One will notice that, in $g_{\nu, 2}$, the mutual coupling terms are not enhanced by resonance.

If, in both solutions, we group the terms with frequencies $\sigma_{\mu}$ and $\sigma_{\nu}$ and, among the others, keep only those reinforced by the commensurability (1), solution (3) becomes

$$
\begin{aligned}
\xi=\xi_{\mu}\left(r_{0}\right)\left\{A _ { \mu } ^ { \prime } \operatorname { c o s } \left(\sigma_{\mu} t+\right.\right. & \left.\left.\Psi_{\mu}^{\prime}\right)-\frac{E}{2 \epsilon \sigma_{\nu}} \cos \left[\left(\sigma_{\mu}+\epsilon\right) t-\Phi\right]\right\} \\
& +\xi_{\nu}\left(r_{0}\right)\left\{A_{\nu}^{\prime} \cos \left[\left(2 \sigma_{\mu}+\epsilon\right) t+\Psi_{\nu}^{\prime}\right]+\frac{d}{8 \epsilon \sigma_{\mu}} \cos 2 \sigma_{\mu} t\right\} .
\end{aligned}
$$

In normal conditions, the lowest mode $\left(\sigma_{\mu}\right)$ is more easily excited than the highest mode $\left(\sigma_{\nu}\right)$, and, correspondingly, the coefficients in the first term are likely to be larger than those in the second term, so that the main beat phenomenon is due to the first term, while the second simply modifies the shape of the oscillation. But the two oscillations of frequencies $\sigma_{\mu}$ and $\sigma_{\mu}+\epsilon$, which, in this theory, are to be identified with the two nearly equal observed frequencies $\sigma$ and $\sigma^{\prime}$, have rigorously the same spatial behavior determined by $\xi_{\mu}\left(r_{0}\right)$. It is thus impossible for them to affect differently the motion of the external layers or the macroturbulence which may prevail there.

Van Hoof tried to avoid this objection by appealing to the behavior of "trial solutions" used by the present author to illustrate the determination of eigen-solutions on a differential analyzer. It is well known that, close to the surface, a trial solution increases much more rapidly than the eigen-solution if the trial period is greater than the eigen- 
period or, alternatively, increases less rapidly and eventually decreases if the trial period is smaller than the eigen-period. Van Hoof identifies the $P^{\prime}$ mode with the fundamental eigen-solution $\left(P^{\prime}=2 \pi / \sigma_{0}\right)$ and the $P$-mode with the oscillation excited by resonant coupling $\left[P=2 \pi /\left(\sigma_{0}+\epsilon\right)\right]$. But he assimilates the spatial behavior of the latter to that of a trial solution with the same period. This would mean that, close enough to the surface, the corresponding amplitude $(\delta r)_{P}$ would increase or decrease very rapidly according to whether $P$ is greater or smaller than $P^{\prime}$. Referring to Struve's table (1955), $(\delta r)_{P}$ would then increase more rapidly than $(\delta r)_{P^{\prime}}$, in all the known $\beta$ Cephei stars except $\beta$ Canis Majoris.

However, these trial solutions have no physical meaning whatever, since they violate the boundary condition $\delta p=0$, at the surface where $(\delta r)_{\text {trial }}$ tends toward $+\infty$ or $-\infty$ and there is no means, either in a linear or in a non-linear theory, to excite physical oscillations exhibiting a spatial behavior similar to that of these trial solutions. Even in the case of a forced oscillation under the action of an external force of frequency $\left(\sigma_{0}+\epsilon\right)$, the variation of its amplitude inside the star will not in any way resemble that of the corresponding trial solution. Thus, for purely radial oscillations, the ordinary coupling theory provides the only possibility of explaining the presence of two very close periods in the observed variation, but, as recalled above, the amplitudes of the two corresponding oscillations will then be practically identical, and there is no explanation for the broadening of the lines associated with the mode $P^{\prime}$. There is also very little prospect that further developments, such as the taking into account of higher-order terms or of more complete series of type (2), will modify this conclusion, although, in that case, other commensurabilities and higher-order subharmonic resonances may acquire some importance.

On the other hand, where coupling plays a dominant role, one must expect that the second term in equation (7), although smaller, will distort the main oscillation, causing appreciable deviations from the sinusoidal shape. But there is no observational evidence for such distortions of the $P$ or the $P^{\prime}$ mode in the $\beta$ Cephei stars.

Furthermore, Detre's remark (1956) in the case of the RR Lyrae stars could be worked out into an empirical test in this case also: the closer the resonance, i.e., the longer the beat period, the greater should be the variation of the amplitude of the primary oscillation in the course of the beat cycle. But again the known data on the semiamplitude $K$ and $K^{\prime}$ of the radial-velocity-curves do not suggest the presence of such a correlation.

\section{ON THE OCCURRENCE OF NEARLY COMMENSURABLE PERIODS}

In the preceding section, we did not specify the two modes presenting the $2: 1 \mathrm{com}$ mensurability. Since, on general grounds, the damping of a mode increases with its order, the coupling theory is most satisfactory when it works with the first two modes, say, of periods $P_{0}$ and $P_{1}$. But for the standard model, taking into account the pressure of radiation which, in these stars, may reduce the generalized adiabatic exponent $\Gamma_{1}$ to a value of the order of 1.55 , we find, interpolating between Schwarzschild's results (1941), $P_{1} / P_{0} \simeq 0.7$, which is very far from the necessary ratio $1: 2$.

It has been shown elsewhere (Elsen and Ledoux 1955; also Lucas 1956) that the existence of an extensive external convection zone tends to reduce $P_{1} / P_{0}$ to a value closer to $\frac{1}{2}$. But, at the same time, this increases the theoretical value of $P_{0} \sqrt{ } \bar{\rho} / \bar{\rho}_{\odot}$, making it more difficult to achieve agreement between theory and observation with respect to the period $P$ (or $P^{\prime}$ ) of the main cycle.

Other modes, such as $P_{2}$ or $P_{3}$, although already less favorable on general grounds, do not present either a sufficiently close commensurability with $P_{0}$. One should not forget that the observations imply a commensurability so close that $|\epsilon|$ in equation (1) should not be greater than $0.02 \sigma_{0}$. This seems a pretty stringent requirement for the model, especially since it must, at the same time, be vibrationally unstable, at least toward the fundamental mode. 


\section{THE EFFECT OF A RADIAL PULSATION ON CONVECTION IN THE EXTERNAL LAYERS}

Although we have seen in Section I that, in the coupling theory, the modes $P$ and $P^{\prime}$ cannot have distinct effects on macroturbulence or convection in the external layers, the general problem of the mutual interaction of pulsation and convection is an important one not only in this context. Van Hoof discusses this point mainly in terms of the temperature gradient, but one should not forget that what matters for convective stability is the difference between the temperature gradient and the corresponding adiabatic gradient, which are both modified by the pulsation.

Let us write

$$
D=\frac{1}{T} \frac{d T}{d r}-\frac{\Gamma_{2}-1}{\Gamma_{2}} \frac{1}{p} \frac{d p}{d r}
$$

so that we have instability if $D<0$ and stability if $D>0$.

In a region where the pulsation can be considered adiabatic, it is easy to verify that

$$
\frac{\delta D}{D}=-\frac{d \delta r}{d r}+\left\{\frac{\partial \log \left[\left(\Gamma_{2}-1\right) / \Gamma_{2}\right]}{\partial \log T}\right\}_{p} \frac{\delta T}{T},
$$

treating $\left(\Gamma_{2}-1\right) / \Gamma_{2}$ as a function of $p$ and $T$. If the second term on the right can be neglected, instability in an initially unstable region is reinforced at contraction and weakened at expansion, provided that $\delta r$ increases with $r$, which is certainly the case everywhere for the fundamental mode and is also true for the first mode in the external region. Furthermore, this effect is stronger, the greater the increase in $\delta r$ with $r$. Thus, even if van Hoof's argument about the trial solution had been correct, his identification of the two modes $P$ and $P^{\prime}$ (cf. Sec. I) would in all stars, except $\beta$ Canis Majoris, lead to a greater variation of macroturbulence and thus of the line profile following the $P$ mode rather than the $P^{\prime}$ mode, which is contrary to the observations.

As far as the last term in equation (9) is concerned, it is not likely, in a B star, to become important anywhere. However, very close to the surface, non-adiabatic and nonlinear terms, although probably smaller than in ordinary cepheids, may still appreciably modify $\delta D / D$ It is not absolutely excluded, either, that the wave could take a progressive character in these external layers, and this also could affect $\delta D / D$. Here we touch one of the sore points of pulsation theory in general: apart from the periods, all the observations refer essentially to the very external layers, where a straightforward extrapolation of the simple pulsation in the interior is not generally justified, although the $\beta$ Cephei stars offer probably one of the cases where this procedure entails less danger.

\section{SOME FURTHER REMARKS ON THE PROBLEM}

Van Hoof's idea of correlating the broadening of the lines with a variation of the macroturbulence related in some way to the $P^{\prime}$ mode is valuable, even if in the present state of the theory of convection it can be developed only qualitatively. It is compatible with Huang's remark that the total equivalent width of the lines remains constant through the stages of broadening or doubling.

It could also account for the van Hoof effect, and, provided that non-linear and (or) non-adiabatic terms come into play at maximum compression to decrease the convection where the amplitude is particularly large, it could perhaps also explain the doubling of the lines. However, in BW Vulpeculae, this would imply a velocity difference between the ascending and descending currents of the order of $120 \mathrm{~km} / \mathrm{sec}$, which is many times the velocity of sound, a result which it would be very difficult to reconcile with any theory of convection.

The observation of double lines has led Struve (1955) and Odgers (1956) to suggest another hypothesis also based on radial pulsations in which a thin layer of the atmosphere is ejected at regular intervals determined by the fundamental period $P_{0}$ of the 
main interior. This layer then falls back under the action of surface gravity. If the time of fall, say $P_{f}$, is smaller than $P_{0}$, there is a standstill on the velocity-curve, as in BW Vulpeculae.

If $P_{f}$ is very slightly greater than $P_{0}$, it is suggested that the interfering motions of the two layers might give the appearance of a beat phenomenon. This is not at all certain, and, moreover, it is practically impossible that $P_{f}$ could be fixed with the necessary precision to insure the regularity of the beat phenomena observed, in some cases, over very long periods of time. The only possibility would be that $P_{f}$ be strictly controlled, as, for instance, in a proper oscillation of the atmosphere.

Although in this form the idea might be fruitful, the difficulty is to explain how the interior and the atmosphere can be made sufficiently independent that their respective proper periods will keep their individual significance. Furthermore, in a B star, one would rather expect that the fundamental period of the atmosphere given in a first approximation by

$$
P_{f}=\frac{2 \pi}{1.2} \sqrt{\frac{h}{\Gamma_{1} g}}
$$

where $h$ is the depth of the atmosphere, would be much smaller than the fundamental period $P_{0}$ of the whole star.

In the author's opinion, non-radial oscillations still retain some definite advantages. First of all, they provide a ready explanation for the fact that in these stars $\Delta m / K$ or $\Delta m^{\prime} / K^{\prime}$ are much smaller than in ordinary intrinsic variables.

Second, in the presence of rotation (Ledoux 1951), they explain in the most natural way the existence of very close frequencies differing by multiples of the angular velocity of rotation $\Omega$. Indeed, in that case, multiple periods are unavoidable. Note that the presence of a weak magnetic field may have the same effect (Ledoux and Simon 1958).

The beat frequency being proportional to $\Omega$, the faster the star rotates, the shorter the beat period or the larger the difference between $P$ and $P^{\prime}$. Excepting $\beta$ Canis Majoris, this is in rather good agreement with the observations as shown by Struve's table (1955), if we keep to the last column its original interpretation in terms of rotational velocities.

In the case of $\beta$ Canis Majoris, to which the theory was originally applied (Ledoux $1951)$, the broadening being associated with the longest period $\left(P^{\prime}>P\right), P^{\prime}$ was to be identified with the wave traveling in the direction opposite to that of rotation. For free oscillations, this led to the wrong sign for the quarter-period shift of the broadening with respect to the $P^{\prime}$ velocity-curve. This difficulty remains for $\beta$ Canis Majoris, but, in all the other cases in Struve's table, $P^{\prime}$ is smaller than $P$ and the $P^{\prime}$ mode can be identified with the wave traveling in the same direction as the rotation giving the right phase shift. Also, the larger amplitude $K^{\prime}$ in all these stars would facilitate the quantitative interpretation of the broadening as compared to the case of $\beta$ Canis Majoris.

Of course, here, also, variations in turbulence could be superposed on the pulsation not only because of the variations of $D$ but also because of the non-uniform velocity field corresponding to the non-radial oscillations, especially the traveling wave. On the other hand, as far as the behavior of the lines is concerned, a non-radial oscillation, with one part of the surface sinking and the other rising, is somewhat comparable to convection and some of the arguments, for instance, in the explanation of the van Hoof effect by convection, could probably be transposed directly to the case of a non-radial oscillation.

Finally, especially in the case of large amplitudes, non-linear effects are bound to arise. In particular, the traveling wave may develop a sharp front which may have something to do with the doubling of the lines.

Of course, in this, as in any other hypothesis, a reliable detailed comparison between 
theory and observations with respect to many of these points could be carried out only after a theoretical discussion of the oscillation in the outer layers had been successfully completed.

\section{REFERENCES}

Detre, L. 1956, Vistas in Astronomy, ed. A. Beer (London: Pergamon Press), 2, 1163.

Elsen, E., and Ledoux, P. 1955, Bull. Soc. R. Sci Liège, 24, 239

Kluyver, H. A. 1936, B. A. N., 7, 313.

Ledoux, P. 1951, Ap. J., 114, 373.

Ledoux, P, and Simon, R., 1957, Ann. d'ap, 20, 185.

Lucas, D. 1956, Bull. Soc. R. Sci. Liège, 25, 585.

Odgers, G. J 1956, Pub. Dom. Ap. Obs. Victoria, 10, 215

Rosseland, S. 1949, The Pulsation Theory of Variable Stars (Oxford: Clarendon Press), Sec 7 7, p 108. Schwarzschild, M. 1941, Ap. J, 94, 245.

Struve, O. 1955, Pub. A S P., 67, 135.

van Hoof, A. 1957, Pub ASP , 69, 308. 